\title{
Feasibility study of an absolute energy calibration of imaging atmospheric Cherenkov telescopes by starlight
}

\author{
O. Karschnick ${ }^{\star}$, H.J. Gils ${ }^{\star \star}$, and W. Stamm \\ Institut für Experimentelle und Angewandte Physik, Leibnizstr. 19, 24118 Kiel, Germany
}

Received December 23, 1999; accepted January 20, 2000

\begin{abstract}
A new method is presented for an absolute energy calibration of imaging atmospheric Cherenkov telescopes as a whole instrument at all relevant wavelengths. For this study the Cherenkov telescope \#1 of the HEGRA $^{1}$ experiment was used. The calibration was carried out by detecting the light of different selected stars during one night. From the data a simple linear correlation was derived between the anode current of the Cherenkov camera photomultiplier tubes and the incident photon flux. This result is important for the imaging air Cherenkov technique in several aspects: The method enables to determine the response function of Cherenkov telescopes in the energetic range of Cherenkov light quickly and reliably. Therefore it can be used as an atmospheric calorimeter by measuring the absolute integrated flux of Cherenkov photons. Moreover, the signal of a point run can be predicted and a simulation of the apparatus response can be carried out more reliably.
\end{abstract}

Key words: telescopes — methods: observational — stars: general - cosmic rays

Send offprint requests to: O. Karschnick,

e-mail: Oliver.Karschnick@desy.de

* Present address: II. Institut für Experimentalphysik der Universität Hamburg, Notkestraße 85, 22607 Hamburg, Germany.

** On leave from Forschungszentrum Karlsruhe, Institut für Kernphysik, Karlsruhe, Germany.

1 The High Energy Gamma Ray Astronomy experiment is operated on La Palma (Canary Island) by the HEGRA collaboration formed by the University of Hamburg, MaxPlanck Institut für Kernphysik, Heidelberg, University of Kiel, University of Madrid, Max-Planck Institut für Physik, Munich, University of Wuppertal and the Yerevan Physics Institute.

\section{Introduction}

With the development of the imaging air Cherenkov technique the Very High Energy (VHE) $\gamma$ ray astronomy has considerably been improved or even been established at all. In this technique Cherenkov light emitted from atmospheric showers is observed by an imaging atmospheric Cherenkov telescope (IACT) with a focal camera consisting of a larger number of small size photomultiplier tubes (PMT) most often building an hexagonal array. In order to interpret the measured data in terms of the characteristic quantities of the primary cosmic ray simulations have to be performed of all the processes involved. Starting from the chosen initial properties of the primary the simulation includes interactions within the atmosphere and the production of secondaries, the emission of Cherenkov light and finally its detection by the telescope. For the last step of the simulation procedure it is necessary to have the most precise knowledge possible of the telescope response function.

The operation of a Cherenkov telescope, however, is influenced by various not easily predictable effects, such as accumulation of dust on the mirrors. Therefore, it is not obvious that the response function of the apparatus can be constructed by simply superimposing theoretical functions of the individual components. An experimental absolute calibration which could easily and often be repeated would considerably improve the interpretation of the measured air shower events. Further on, since the Cherenkov light represents the calorimetric component of an electromagnetic shower the integrated absolute flux of the Cherenkov photons is an important parameter the exact knowledge of which leads to a considerable improvement of the interpretation of the measurement.

For an absolute calibration a simple procedure has been developed based on the detection of light emitted by selected stars having a certain spectrum. Some advantages of this procedure are obvious: starlight can always be detected during clear nights, the method is very cheap and only a small amount of operation time is necessary. 
As compared to other calibration procedures (Fraß et al. 1997; Konopelko et al. 1996; Vacanti et al. 1994) the advantage of the use of starlight as light source is the possibility to calculate a ADC counts to Cherenkov photons conversion factor that is valid in the whole spectral range of Cherenkov light and not only for preselected wavelengths. Additionally, it can be used for small single IACTs.

As an important experimental precondition for this procedure the PMTs of the camera must have an anode current readout with well known features which was especially designed for IACT\#1 (Njoo 1995).

In the present paper the main results of a feasibility study are presented, All further details can be found in Karschnick (1996).

It should be noted that it was not in the scope of this study to develop the method as a mature tool for a routine application. The purpose of the paper is to show that it is worthwhile to invest further research in order to reach this goal.

\section{Experimental set-up and method}

The measurements reported here have been made with the prototype telescope \#1 of the HEGRA collaboration. This telescope has a two-axis equatorial drive head and a $5 \mathrm{~m}^{2}$ segmented mirror plane. In the focal plane, at approximately $5 \mathrm{~m}$ distance, an imaging Cherenkov camera is located. The camera consists of 127 closely packed EMI9083-photomultiplier tubes coupled to light collecting aluminum cones forming a hexagon. The signal at the anode of the photomultiplier tubes is read out by independent electronic circuits, one for direct current (dc-branch) and one for pulse measurements (ac-branch): The output of the dc-branch is equivalent to the dc-anode current with a resolution of 8 bit corresponding to a value of $^{2} I_{\mathrm{LSB}}=0.195 \mu \mathrm{A}$. The output of the ac-branch corresponds to the pulse charges at the anode integrated over $30 \mathrm{~ns}$ and amplified with a factor of $16 \pm 0.5$. The resolution is 12 bit corresponding to $Q_{\mathrm{LSB}}=0.25 \mathrm{pC}$. Hence the errors of the current and charge measurements $\Delta I_{\mathrm{Q}}$ and $\Delta Q_{\mathrm{Q}}$ due to the $\mathrm{ADC}$ quantization are given by $\Delta I_{\mathrm{Q}}=$ $I_{\mathrm{LSB}} / 2= \pm 0.097 \mu \mathrm{A}$ and $\Delta Q_{\mathrm{Q}}=Q_{\mathrm{LSB}} / 2= \pm 0.125 \mathrm{pQ}$ (Tietze \& Schenk 1991). Other uncertainties such as temperature drifts have been found to be negligible compared to these figures in careful laboratory tests. More details can be found in Mirzoyan (1995) and Njoo (1995).

The most important consideration of the method is, what kind of stellar types have to be selected in order to obtain an emission spectrum and detection sensitivity similar to Cherenkov light. Telescope \#1 is especially sensitive in the wavelength range of approximately $260 \mathrm{~nm}$ to $650 \mathrm{~nm}$. The observed energy spectrum of photons hitting the telescope is determined by the primary photon

\footnotetext{
${ }^{2}$ LSB $=$ Least Significant Bit.
}

spectrum corrected for the extinction in the atmosphere. A simulation of Cherenkov light production by cosmic rays and its extinction was performed by Akhperjanian \& Wiedner (1993) and their results have been adopted for the present study. Since the Cherenkov light of an air shower induced by a $\mathrm{VHE} \gamma$ ray does not traverse the entire atmosphere, owing to the fact that most of it is emitted below the ozone layer, the extinction is less than for starlight. Nevertheless, the spectrum of detected Cherenkov light is very steep, as can be seen in Fig. 1.

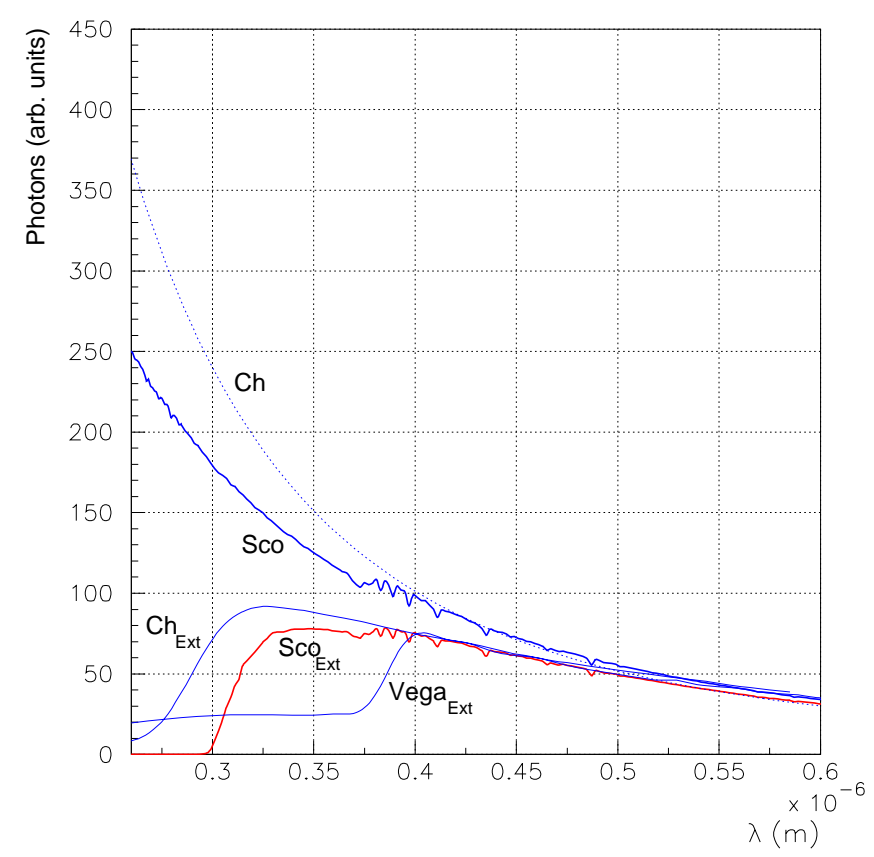

Fig. 1. Spectra of Cherenkov light ("Ch") and of $\tau$ Scorpius ("Sco", approximately $30000 \mathrm{~K}$ ) with extinction in the atmosphere (index "Ext") and without. As an example of a spectrum not to be used for this method, the spectrum of Vega ("Vegaext", approximately $9500 \mathrm{~K}$ ) is also plotted. All spectra are normalized at $\lambda=0.55 \mu \mathrm{m}$

For comparison, different stellar spectra are plotted in Fig. 1 as well. These spectra are calculated by using simulations for stellar atmospheres performed by Kurucz (Kurucz 1979, 1993; Kurucz et al. 1974), which gave very realistic results when compared to actual measured spectra (Malagnini 1983; Longo et al. 1989). The flux at the top of the Earth's atmosphere is calculated in terms of $U B V$ photometry (Hagen \& Boksenberg 1995). The atmospheric extinction on La Palma was calculated within reasonable models based on the most important effects such as Rayleigh and Mie scattering as well as molecular Ozone absorption. These model calculations were adjusted to measurements of the (close by) Carlsberg Observatory.

Although no stellar spectrum observed at the Earth fits the Cherenkov light spectrum perfectly, very hot stars which do not show distinct absorption bands in the 
relevant wavelength range do approximate it quite well. Using the Pogson formula (Budding 1993) the calculation of color indices of stellar spectra that do so is easy. Stars with color indices of about $B-V=-0$. 105 and $U-B=-1$. 730 would fit the plotted spectrum of Cherenkov light in Fig. 1 (Karschnick 1996).

Two other points have to be considered when the various stars are selected. First, the stars have to be bright enough and second, the stars have to be visible under small zenith angles in order to keep the fluctuation of the atmospheric extinction negligible.

A number of stars, that approximately meet the above criteria, have been selected for the calibration and are given in Table 1. Better candidates were not available for La Palma at that observation time. Since detailed spectral information is not available for most of the stars and in order to treat all stars on equal footings calculated energy and photon fluxes, respectively, based on the simulations of Kurucz $(1979,1993)$ have been used.

The calculated spectra have been normalized in such a way that the energy flux at $\lambda_{u}=550 \mathrm{~nm}$ corresponds to the visual magnitude, thus neglecting the interstellar extinction. The atmospheric extinction, also measured by the Carlsberg Observatory at $\lambda_{u}=550 \mathrm{~nm}$ in the same night when the starlight measurements were made, have been extrapolated to the sensitive wavelength region of the telescope $(260 \mathrm{~nm}-650 \mathrm{~nm})$ by the method described above.

\section{Measurements}

The measurement lasted for approximately 15 minutes for each star observed. The principle of the measurements is called point run which is normally used as an angular adjusting procedure for the telescope. For such a point run a certain area of the camera is covered with a prechosen virtual point grid. On each coordinate of this grid the star is subsequently focused and the anode current of each photomultiplier is read out and recorded. At the beginning and at the end of such runs, the central pixel is pointed to the star.

In this pilot study stars were only projected onto approximately 15 photomultiplier tubes of the central region of the camera. The grid had a dimension of $15 \times 15$ points. Covering the whole camera surface by the calibration procedure is a trivial problem of more measuring time.

The selected stars (see Sect. 2) which have been used and some characteristic data are listed in Table 1.

\section{Data analysis}

The analysis of the point run data undergoes various phases for determining the final experimental values of the anode currents.
1. Detection of the star's image in the camera plane. Considering the geometry of the camera and the size of the star's image at most three PMTs could be hit;

2. Detection of background currents in the PMTs surrounding the image area to be appropriately subtracted from the star's current;

3. Rejection of events where the star's picture could not be reconstructed. A typical of such event is a signal in a string of three PMTs whereas doublet and triplet clusters are allowed;

4. Determination of the mean currents from the grid points.

The energy flux and the photon current, respectively, at the telescope mirrors before being reflected have been calculated as described above. For these calculations an error of $10 \%$ has been deduced for the energy flux above the atmosphere (Torres 1987), of $2 \%$ for the extinction (Carter \& Clegg 1994; Hayes et al. 1975) and of $1 \%$ for the $z$-angle dependency during the measurement yielding in an error of $10.2 \%$. The theoretical anode currents are then calculated in a straight forward way considering the known characteristics of the different parts of the telescope, i.e. spectral reflectivity of the mirrors and the aluminum cones, spectral quantum efficiency and mean gain of the PMTs. This step of the calculation incorporates an error of $8.4 \%$ which is typical for the apparatus used. Considering all uncertainties in the calculation of the theoretical anode current a total error of approximately $13 \%$ is determined.

\subsection{Direct current analysis}

In Fig. 2 (top graph) the measured mean direct anode currents of each star $I_{\mathrm{m}}$ is plotted versus the theoretically expected currents $I_{\text {th }}$.

The errors assigned to the measured currents arise from statistics in the grid points and from the systematic uncertainties due to the background brightness in the surrounding pixels (see above). In spite of the various assumptions and uncertainties the data points represent very nicely a linear relationship. Using a linear least squares fit one obtains

$$
I_{\mathrm{th}}=0.775( \pm 0.088) I_{\mathrm{m}}+0.301( \pm 0.444) \mu \mathrm{A} .
$$

It can be concluded that within the limited accuracy of the method the direct anode current is a linear measure for the photon flux of stars detected by the IACT.

\subsection{Transient response for pulse operation}

For the final goal of energy calibration of IACTs, the response function has to be transferred to pulse operation. The necessary general capability of the electronic circuits is given as pointed out before. For purposes of convenience and tracing the procedure the measured anode current is 
Table 1. Stars the photon flux of which is measured for the calibration. Given are their HR number, locii (right ascension, declination), spectral type (taken out of the Astronomical Almanac, Hagen \& Boksenberg 1995, and only for 68 Her and 12 Vul from Friedrichsen 1993) and their effective temperature (estimated with Lang 1992). 10 Lac was measured twice. We are aware that the spectral types and absolute magnitudes of the chosen stars are slightly variable. This can in principle be considered by forthcoming applications of the method but was not in the scope of the present study

\begin{tabular}{cccccccc}
\multicolumn{2}{c}{\begin{tabular}{c} 
Flamsteed/Bayer \\
\multicolumn{2}{c}{ Designation }
\end{tabular}} & HR No. & $\begin{array}{c}\text { Right } \\
\text { Ascension }\end{array}$ & Declination & $\begin{array}{c}\text { Spectral } \\
\text { Type }\end{array}$ & $\begin{array}{c}\text { Effective } \\
\text { Temperature }\end{array}$ \\
\hline 4 & $\theta$ & CrB & 5778 & $15^{\mathrm{h}} 32^{\mathrm{m}} 44.9^{\mathrm{s}}$ & $+31^{\circ} 22^{\prime} 27^{\prime \prime}$ & B6 Vnn & $\sim 14000 \mathrm{~K}$ \\
22 & $\tau$ & Her & 6092 & $16^{\mathrm{h}} 19^{\mathrm{m}} 36.3^{\mathrm{s}}$ & $+46^{\circ} 19^{\prime} 26^{\prime \prime}$ & B5 IV & $\sim 15000 \mathrm{~K}$ \\
68 & & Her & 6431 & $17^{\mathrm{h}} 17^{\mathrm{m}} 19.4^{\mathrm{s}}$ & $+33^{\circ} 06^{\prime} 00^{\prime \prime}$ & B3 III & $\sim 17000 \mathrm{~K}$ \\
85 & $\iota$ & Her & 6588 & $17^{\mathrm{h}} 39^{\mathrm{m}} 20.3^{\mathrm{s}}$ & $+46^{\circ} 00^{\prime} 31^{\prime \prime}$ & B3 IV & $\sim 19000 \mathrm{~K}$ \\
67 & & Oph & 6714 & $18^{\mathrm{h}} 00^{\mathrm{m}} 25.2^{\mathrm{s}}$ & $+02^{\circ} 55^{\prime} 53^{\prime \prime}$ & B5 Ib & $\sim 13500 \mathrm{~K}$ \\
102 & & Her & 6787 & $18^{\mathrm{h}} 08^{\mathrm{m}} 34.0^{\mathrm{s}}$ & $+20^{\circ} 48^{\prime} 49^{\prime \prime}$ & B2 IV & $\sim 21000 \mathrm{~K}$ \\
10 & $\beta$ & Lyr & 7106 & $18^{\mathrm{h}} 49^{\mathrm{m}} 54.8^{\mathrm{s}}$ & $+33^{\circ} 21^{\prime} 26^{\prime \prime}$ & B7 Vpe (shell) & $\sim 13000 \mathrm{~K}$ \\
20 & $\eta$ & Lyr & 7298 & $19^{\mathrm{h}} 13^{\mathrm{m}} 36.3^{\mathrm{s}}$ & $+39^{\circ} 08^{\prime} 17^{\prime \prime}$ & B2.5 IV & $\sim 19000 \mathrm{~K}$ \\
8 & & Cyg & 7426 & $19^{\mathrm{h}} 31^{\mathrm{m}} 36.3^{\mathrm{s}}$ & $+34^{\circ} 26^{\prime} 36^{\prime \prime}$ & B3 IV & $\sim 18000 \mathrm{~K}$ \\
12 & & Vul & 7565 & $19^{\mathrm{h}} 51^{\mathrm{m}} 04.0^{\mathrm{s}}$ & $+22^{\circ} 36^{\prime} 36^{\prime \prime}$ & B2.5 Ve & $\sim 19000 \mathrm{~K}$ \\
59 & $\mathrm{v} 832$ & Cyg & 8047 & $20^{\mathrm{h}} 59^{\mathrm{m}} 40.4^{\mathrm{s}}$ & $+47^{\circ} 30^{\prime} 12^{\prime \prime}$ & B1.5 Vnne & $\sim 23000 \mathrm{~K}$ \\
66 & & Cyg & 8146 & $21^{\mathrm{h}} 17^{\mathrm{m}} 44.0^{\mathrm{s}}$ & $+34^{\circ} 52^{\prime} 40^{\prime \prime}$ & B2 Ve & $\sim 22000 \mathrm{~K}$ \\
10 & & Lac & 8622 & $22^{\mathrm{h}} 39^{\mathrm{m}} 03.5^{\mathrm{s}}$ & $+39^{\circ} 01^{\prime} 36^{\prime \prime}$ & O9 V & $\sim 33000 \mathrm{~K}$ \\
81 & $\pi^{2}$ & Cyg & 8335 & $21^{\mathrm{h}} 46^{\mathrm{m}} 37.6^{\mathrm{s}}$ & $+49^{\circ} 17^{\prime} 19^{\prime \prime}$ & B2.5 III & $\sim 18000 \mathrm{~K}$ \\
\hline
\end{tabular}

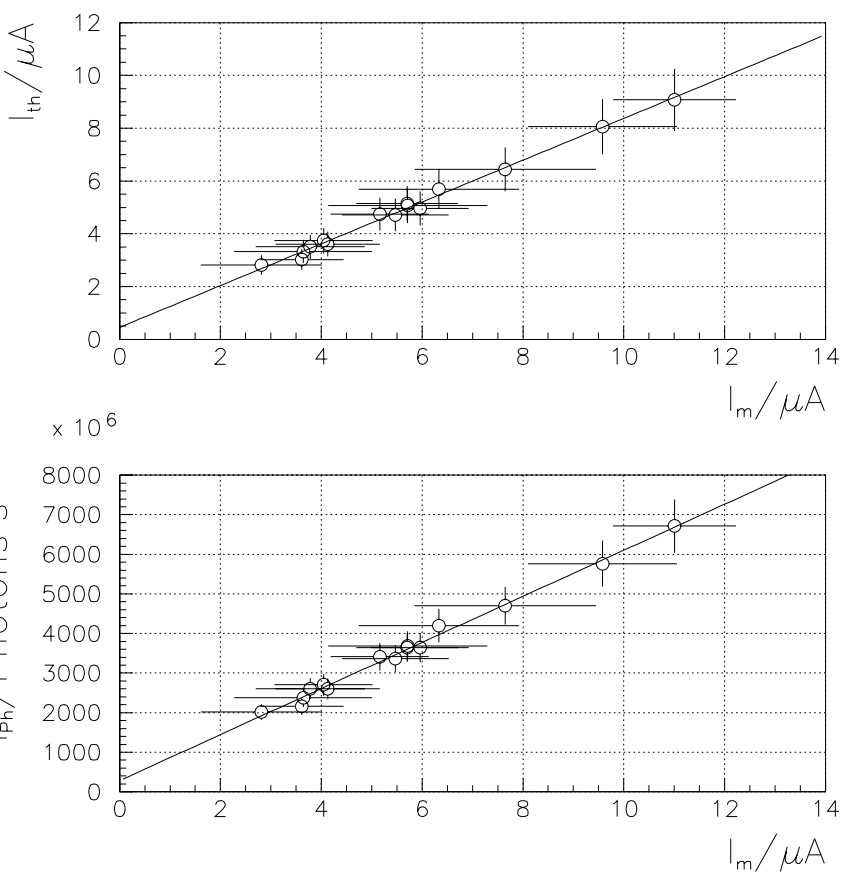

Fig. 2. Calculated anode currents $I_{\text {th }}$ (top graph) and incident photon current $I_{\mathrm{Ph}}$ (bottom graph) of the stars given in Table 1 versus the measured dc currents $I_{\mathrm{m}}$. A linear least square fit is plotted for both distributions

first related with the calculated energy flux. This is very helpful when selecting stars for angular calibration point runs. A compilation of predicted anode currents for various stellar parameters is given in Karschnick (1996).

In the second step the mirror area and the spectral energy flux have to be considered to calculate the spectral photon current $I_{\mathrm{Ph}}$. This quantity is plotted in the bottom graph of Fig. 2. The parameters of the linear correlation are given by

$$
\begin{aligned}
I_{\mathrm{Ph}}=\frac{5.82( \pm 0.49) 10^{14}}{C} & I_{\mathrm{m}} \\
& +2.82( \pm 2.33) 10^{8} \frac{\text { Photons }}{\mathrm{s}} .
\end{aligned}
$$

When detecting pulses of Cherenkov light, the readout electronics sample the charge at the anode $Q_{\text {Anode }}$ with an integration time of 30 ns leading to the number of the incident photons $N_{\mathrm{Ph}}$ given by

$$
N_{\mathrm{Ph}}=\frac{5.8210^{14}}{C} \cdot Q_{\text {Anode }}
$$

where the offset is neglected (see below). The error of $N_{\mathrm{Ph}}$ deduced from Eq. (2) is approximately $8 \%$. The additional error introduced to the pulse branch by considering the offset is negligible. For the Q-ADC the gain of this branch has finally to be considered, resulting in a calibration function for pulse operation:

$$
N_{\mathrm{Ph}}=\frac{5.8210^{14}}{C} \cdot \frac{Q_{\mathrm{Q}}}{16} .
$$

If, for example, $1 \mathrm{pC}$ is read out at the PMT, corresponding to 4 channels, approximately 36 photons have reached the telescope. The maximum value of $256 \mathrm{pC}$ corresponds to about 9300 incident photons.

\section{Discussion}

With the calibration procedure the response of the whole IACT was found to be approximated very well by a linear 
function. The errors of the method are sufficiently and can be improved by further studies.

An estimate for one flash of Cherenkov light using Eq. (2) leads to approximately 10 photons from the night sky background. Taking mean values for the mirror and cone reflectivity, quantum efficiency and photomultiplier gain this corresponds to 1 photoelectron. This figure matches very well with other considerations (Krennrich 1995).

The small offset of the calibration function is not fully understood yet. It may be based on the night sky background and its fluctuation. This contribution could not be included in the background subtraction described in Sect. 4 because it is not measured (threshold effect). Including this into the data analysis would perfectly remove the offset.

The main result (Eq. 4) can be compared with the measured photoelectron to ADC-count conversation factor from Mirzoyan et al. (1995). With his result (i.e. 1 photoelectron corresponds to $1.39( \pm 10 \%)$ ADC channels $)$ using mean values for the mirror reflectivity (85\%), the quantum efficiency (12\%) and the loss at the aluminum cones of the photomultiplier $(11 \%)$ the absolute calibration is compatible. A vulnerable proof of the calibration would be if this method and another independent one is applied to the same telescope.

The results achieved here can reach more accuracy by measuring various stellar spectra and the atmospheric extinction parallel to the calibration measurements. Nevertheless, these measurements show the possibility of absolute calibration by means of a very simple method which, therefore, is planned to be used in new IACT-projects (Aharonian et al. 1997).

Acknowledgements. We would like to thank all members of the HEGRA collaboration for their support during that work especially H.G. Börst, G. Heinzelmann and C.A. Wiedner for fruitful discussions as well as M. Hemberger, A. Konopelko and R. Mirzoyan who realized the measurements with the Cherenkov telescope \#1. H.J.G. acknowledges the kind hospitality received by IEAP during his stay at the University of Kiel.

\section{References}

Akhperjanian A., Wiedner C.-A., 1993, Detection of Cerenkov Flashes with the CT-Telescopes at La Palma, Ct - Note 15, HEGRA-collaboration
Aharonian F., Daum A., Hermann G., et al., 1997, HESS letter of intent, Max-Planck-Institut für Kernphysik, Heidelberg and II. Institut für Experimentalphysik, Universität Hamburg, Germany

Budding E., 1993, An Introduction to Astronomical Photometrie. Cambridge University Press. Cambridge, U.S.A.

Carter D., Clegg R.E.S., 1994, Observers' Guide. Isaac Newton Group La Palma

Fraß A., Köhler C., Hermann G., et al., 1997, Astropart. Phys. 8, 91-99

Friedrichsen T.R., 1993, Xsky Sternenatlas, Version 2.0.1, in: S.u.S.E. Linux April 1995, CDRom 1, S.u.S.E. GmBH, Fürth, Germany

Hagen J.B., Boksenberg A., 1995, Astronomical Almanac, U.S. Government Printing Office

Hayes D.S., Latham D.W., 1975, ApJ 197, 593

Karschnick O., 1996, Absoluteichung von CherenkovTeleskopen mit Sternenlicht, Diploma Thesis, Institut für Reine und Angewandte Kernphysik Kiel, Germany (unpublished)

Konopelko A., Aharonian F., Akhperjanian A., et al., 1996, Astropart. Phys. 4, 199

Krennrich F., 1995, Beobachtungen von $\mathrm{TeV}$ Photonen aus der Richtung des Krebsnebels mit dem $5 \mathrm{~m}^{2}$ Tscherenkovteleskop des HEGRA Projektes, Ph. Thesis, Max-Planck-Institut für Physik, München, Germany

Kurucz R.L., Peytremann E., Avrett E.H., 1974, Blanketed Model Atmospheres for Early Type Stars, Smithonian Institute, Washington DC, U.S.A.

Kurucz R.L., 1979, ApJS 40, 1

Kurucz R.L., 1993, CD ROM 13, ATLAS9 Stellar Atmospheres Programs and $2 \mathrm{~km} \mathrm{~s}^{-1}$ Grid, Smithonian Astrophysical Observatory, Cambridge, U.S.A.

Lang K.R., 1992, Astrophysical Data: Planets and Stars. Springer Verlag, New York, U.S.A.

Longo R., et al., 1989, ApJ 339, 474

Malagnini M.L., 1983, A\&A 128, 375

Mirzoyan R., Fernandez J., Lorenz E., et al., 1995, Proc. Int. Workshop on TeV Gamma-Ray Astrophysics, Towards a Major Atmospheric Cherenkov Detektor IV, Padova, Italy, M. Cresti (ed.), p. 230-240

Njoo K.P., 1995, Untersuchungen an Diskriminatoren für eine hochauflösende Cherenkov-Kamera Diploma Thesis, Institut für Reine und Angewandte Kernphysik Kiel, Germany (unpublished)

Tietze U., Schenk C., 1991, Halbleiter Schaltungstechnik. Springer Verlag, Berlin, Heidelberg, New York

Torres A.V., 1987, ApJ 322, 949

Vacanti G., Fleury P., Jiang Y., et al., 1994, Astropart. Phys. 2,1 